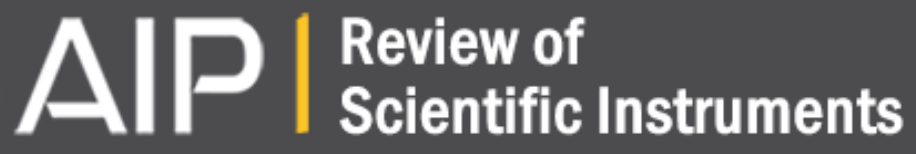

\section{Microwave-induced acoustic imaging of biological tissues}

Lihong V. Wang, Xuemei Zhao, Haitao Sun, and Geng Ku

Citation: Review of Scientific Instruments 70, 3744 (1999); doi: 10.1063/1.1149986

View online: http://dx.doi.org/10.1063/1.1149986

View Table of Contents: http://scitation.aip.org/content/aip/journal/rsi/70/9?ver=pdfcov

Published by the AIP Publishing

\section{Articles you may be interested in}

X-ray acoustic computed tomography with pulsed $\mathrm{x}$-ray beam from a medical linear accelerator

Med. Phys. 40, 010701 (2013); 10.1118/1.4771935

Near-field radiofrequency thermoacoustic tomography with impulse excitation

Med. Phys. 37, 4602 (2010); 10.1118/1.3467756

Magnetoacoustic tomography with magnetic induction for imaging electrical impedance of biological tissue J. Appl. Phys. 99, 066112 (2006); 10.1063/1.2186371

Signal processing in scanning thermoacoustic tomography in biological tissues Med. Phys. 28, 1519 (2001); 10.1118/1.1380436

Acoustic nonlinearity parameter tomography for biological tissues via parametric array from a circular piston source-Theoretical analysis and computer simulations

J. Acoust. Soc. Am. 109, 1219 (2001); 10.1121/1.1344160

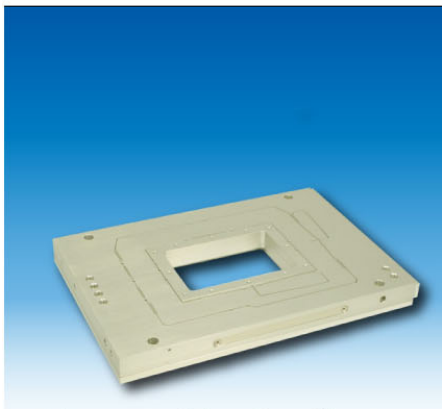

Nanopositioning Systems

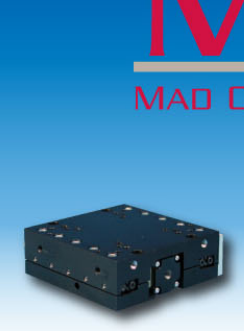

Micropositioning

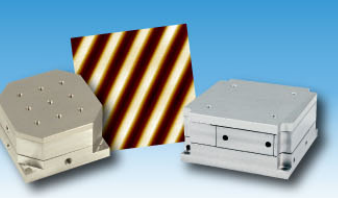

AFM \& SPM

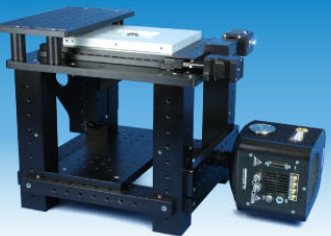

Single molecule imaging 


\title{
Microwave-induced acoustic imaging of biological tissues
}

\author{
Lihong V. Wang, Xuemei Zhao, Haitao Sun, and Geng Ku \\ Optical Imaging Laboratory, Biomedical Engineering Program, Texas A\&M University, College Station, \\ Texas 77843-3120
}

(Received 19 March 1999; accepted for publication 15 June 1999)

\begin{abstract}
We present tomographic imaging of biological tissues by use of microwave-induced acoustic signal. It was demonstrated that the acoustic signal was proportional to the intensity of the incident microwave and was related to the absorption property of microwave in the medium. Pulsed microwave radiation was used to illuminate the samples. Absorbed microwave energy caused thermoelastic expansion that radiated acoustic waves. A focused ultrasonic transducer detected the time-resolved acoustic signals. Each acoustic signal was converted into a one-dimensional image. A linear scanning of the ultrasonic transducer yielded multiple one-dimensional images, which formed a two-dimensional image. The imaging contrast is based on the difference in the dielectric constants among biological tissues. Because of the large contrast in microwave absorption among different tissue types, microwave-induced acoustic tomography could potentially provide a new modality for detecting early-stage cancers. (C) 1999 American Institute of Physics. [S0034-6748(99)04709-7]
\end{abstract}

\section{INTRODUCTION}

Microwave imaging of biological tissues has been investigated for a number of years. ${ }^{1-5}$ The advantages of the technique include the use of nonionizing radiation and high imaging contrast. However, the technique has had difficulties in multichannel detection of microwave without cross coupling, in the reconstruction algorithms, and in achieving good spatial resolution. Ultrasound imaging (ultrasonography), an established medical imaging modality, can yield good spatial resolution but has poor contrast. Microwave-induced acoustics (MIA) may bridge the gap and combine the advantages of the two types of radiation.

The phenomenon that pulsed microwave generates acoustic signals in lossy media has been recognized since long ago. This phenomenon was called microwave auditory or MIA. MIA was used to quantify physical parameters in media such as the power density and the concentration of a given substance. ${ }^{6-8}$ MIA was also employed by several investigators in the 1980s for imaging of biological tissues or tissue phantoms. ${ }^{9-14}$ However, these early works did not produce any tomographic or depth-resolved images. Recently, images of biological tissue were reconstructed based on MIA. ${ }^{15-18}$ This approach requires measurements of a large amount of data around the tissue and expensive computation following the data acquisition.

Microwave-induced acoustic imaging (MIAI) is based on the detection of the acoustic signals generated by microwave in the samples. Pulsed microwave radiation is used to illuminate the samples. Absorbed microwave energy causes thermoelastic expansion that radiates acoustic waves. An ultrasonic transducer or an array detects the time-resolved acoustic signals. Although MIAI shares similar principles with the photoacoustic imaging in the optical-wavelength region, ${ }^{19-22}$ MIAI may have broader applications in medical imaging because microwave has a greater contrast and a deeper penetration depth in biological tissues than light.
The electric field strength in a lossy media is attenuated exponentially as

$$
E=E_{0} \exp (-\alpha z),
$$

where $E_{0}$ is the electrical field at the sample surface, $E$ is the electrical field at the depth $z$, and $\alpha$ is the electric-field absorption coefficient expressed as

$$
\alpha=\omega \sqrt{\frac{\mu \varepsilon}{2}\left(\sqrt{1+\left(\frac{\sigma}{\omega \varepsilon}\right)^{2}}-1\right)},
$$

where $\omega$ is the angular frequency, $\mu$ is the permeability, $\varepsilon$ is the permittivity, and $\sigma$ is the conductivity. The induced acoustic pressure is expected to depend on the intensity of microwave and the complex dielectric constant of the material. In the frequency range of $0.1-10 \mathrm{GHz}$, the dielectric constant (ratio of the permittivity in material to that in vacuum) has a value of 5-70 for soft tissues, and the conductivity has a value of $0.02-3 \mathrm{~s} / \mathrm{m}^{23-25}$ The dielectric properties of tissue determine the absorption of microwave at various frequencies of microwave. At $9.4 \mathrm{GHz}$, the penetration depths, which are the inverse of the absorption coefficients, are 3.4 and $0.35 \mathrm{~cm}$ for fat and muscle, respectively; while at $500 \mathrm{MHz}$, the penetration depths for fat and muscle are 23.5 and $3.4 \mathrm{~cm}$, respectively. ${ }^{26}$ Most of the other soft tissues have an absorption coefficient in between those of muscle and fat. This wide range of values can provide an extremely high imaging contrast for biological tissues. ${ }^{2}$ Therefore, MIAI may potentially be used to detect earlystage cancers.

We here present our study on MIAI toward biomedical applications. First, some basic properties of MIA were examined using various tissue phantoms. Then, twodimensional cross sections of biological tissues were imaged using MIAI. Our imaging approach differs significantly from the prior arts in MIAI. Lateral resolution was achieved by use of a focused ultrasonic transducer. Axial resolution was 


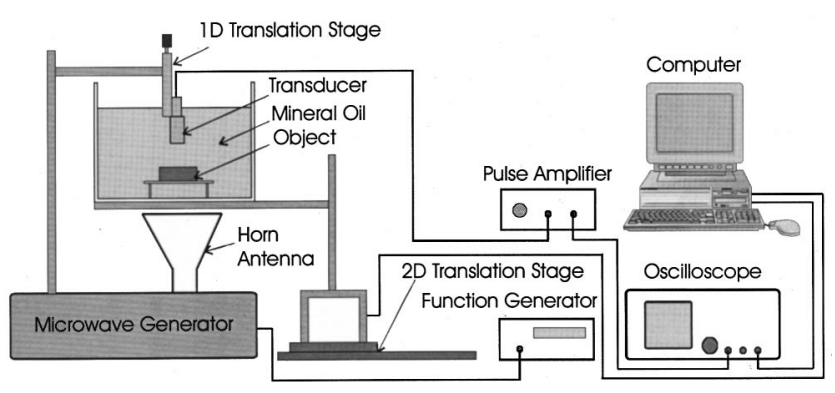

FIG. 1. Experimental setup.

obtained by measuring the temporal profiles of the microwave-induced acoustic signals. Depth-resolved tomographic images were acquired directly without resorting to image reconstruction.

\section{EXPERIMENTAL SETUP}

The experimental setup used for this study is shown in Fig. 1. A Cartesian coordinate system was set up for reference. The $z$ axis was along the ultrasonic axis pointing upward. The $x$ axis was perpendicular to the drawing plane and pointed outward. The $y$ axis was in the drawing plane pointing to the right. A 9.4-GHz microwave generator transmitted microwave pulses of $10-\mathrm{kW}$ peak power into the mineral oil bath through an $X$-band 16-dB horn antenna. The aperture of the horn antenna was $55 \mathrm{~mm}$ long and $74 \mathrm{~mm}$ wide. The pulse width of microwave was set to either 2.2 or $0.6 \mu \mathrm{s}$ in our experiments. A function generator (DS345, Stanford Research System) was employed to trigger the microwave generator and control its pulse repetition frequency. The mineral oil bath was contained in a Plexiglas container and was fixed on a two-dimensional $x-y$ translation stage (MD2, Arrick Robotics). A personal computer controlled the two stepper motors to drive the translation stage in the $x$ and $y$ directions. The object to be scanned was put on a Plexiglas stand inside the bath. Mineral oil has a very small absorption coefficient for microwave and allowed good acoustic coupling. Plexiglas has low microwave absorption as well. An ultrasonic transducer was immersed in the mineral oil facing the microwave antenna. Two ultrasonic transducers were used in this study. For the first one (V314, Panametrics), the central frequency of the ultrasonic transducer was $1 \mathrm{MHz}$, the bandwidth was $0.65 \mathrm{MHz}$, the diameter was $1.9 \mathrm{~cm}$, and the focal length at $1 \mathrm{MHz}$ was $2.5 \mathrm{~cm}$. For the second one (V384, Panametrics), the central frequency was $3.5 \mathrm{MHz}$, the bandwidth was $2.5 \mathrm{MHz}$, the diameter was $0.64 \mathrm{~cm}$, and the focal length at $3.5 \mathrm{MHz}$ was $1.8 \mathrm{~cm}$. The transducer was connected to a pulse amplifier (500PR, Panametrics). The amplified signal was recorded by an oscilloscope (TDS-640A, Tektronix) and transferred to a personal computer.

\section{RESULTS}

In order to study the dependence of the acoustic pressure on the microwave power, we tested a gel made of $5 \%$ gelatin and $95 \%$ water. The gel was a slab with a thickness of $8 \mathrm{~mm}$ and an area much greater than the receiving surface of the ultrasonic transducer. The gel was buried in the mineral oil

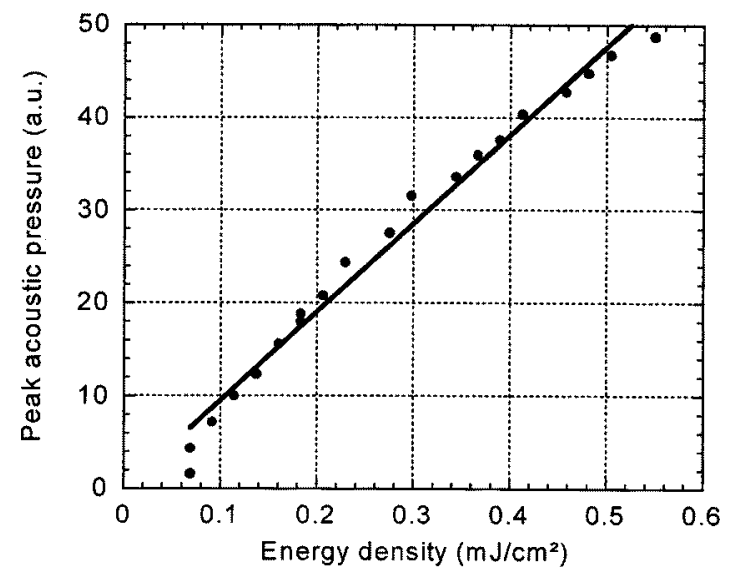

FIG. 2. Dependence of the microwave-induced acoustic pressure on the microwave energy density per pulse. The test sample was an 8-mm-thick gel made of $5 \%$ gelatin and $95 \%$ water.

bath. The acoustic pressure versus the input energy density is plotted in Fig. 2. The input energy density was varied by adjusting the cathode current of the magnetron (M602, Toshiba) in the microwave generator. The result shows that the acoustic pressure increased linearly with the input density as expected.

In Fig. 3, the time-domain acoustic waveforms induced by the microwave in its full power and $12 \%$ of the full power are compared. The full-power microwave had a peak power of $10 \mathrm{~kW}$ and an energy density of $0.55 \mathrm{~mJ} / \mathrm{cm}^{2}$. The $12 \%$ reduced source power yielded a surface energy density equivalent to the energy density at the penetration depth when the sample was illuminated with the full power. The signal-to-noise ratio at the penetration depth was expected to be about 3:1 when the full-power microwave was used in our system based on this measurement. This type of experiments may be used for selecting necessary microwave energy density in MIAI applications.

Three gel slabs were made of the same amount of gelatin $(5 \%)$ and different amounts of $\mathrm{NaCl}(0 \%, 3 \%$, and 5\%). The dimensions of the slabs were 30,30 , and $10 \mathrm{~mm}$ in the $x, y$, and $z$ directions, respectively. The ultrasonic transducer was scanned across each of the slabs along the $x$ direction while

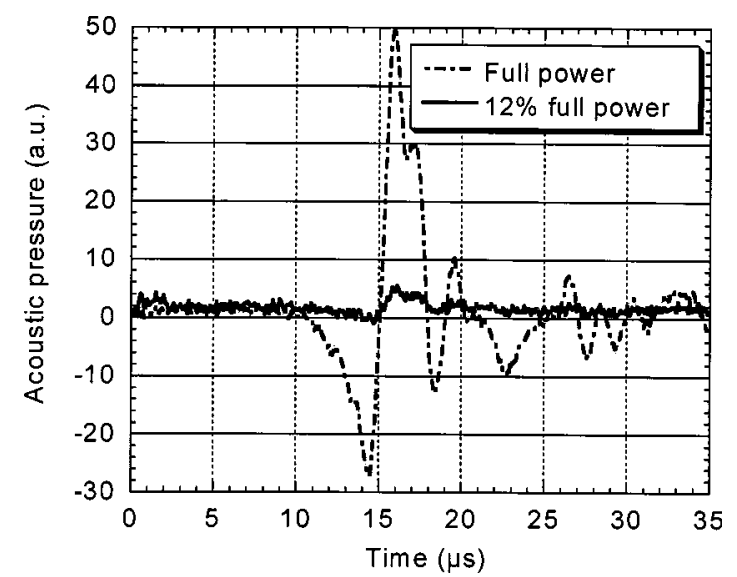

FIG. 3. Waveforms of the microwave-induced acoustic pressure when the sample was exposed to the full power of the microwave generator $(0.55$ $\mathrm{mJ} / \mathrm{cm}^{2}$ ) and to $12 \%$ of the full power. 


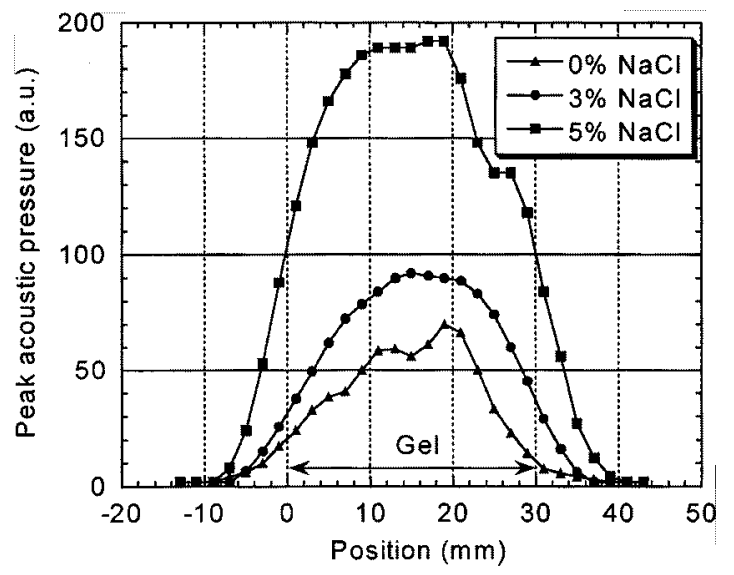

FIG. 4. One-dimensional images of the gel slabs that contain $0 \%, 3 \%$, and $5 \% \mathrm{NaCl}$, respectively. The slabs were $30 \mathrm{~mm}$ wide and $10 \mathrm{~mm}$ thick, located between 0 and $30 \mathrm{~mm}$ on the horizontal axis.

the microwave-induced acoustic signals were recorded. Figure 4 shows the scanned one-dimensional images based on the peak amplitude of the acoustic signals from the bottom surface of the slabs. The peak amplitude increased with increased $\mathrm{NaCl}$ concentration because increasing $\mathrm{NaCl}$ led to an increase in the conductivity of microwave. According to Eq. (2), the energy absorbed by the gel would increase with an increased conductivity and result in an increased acoustic amplitude. These three samples had similar values of acoustic impedance. This experiment demonstrated the contrast mechanism of MIAI compared with that of the conventional ultrasound imaging. MIAI depends on the difference in dielectric constant among different tissue types or materials, while ultrasound imaging depends on the difference in acoustic impedance. Therefore, MIAI has potential to image objects that are invisible with ultrasound imaging.

To examine soft tissue contrast, we scanned in the $x-y$ plane a tissue sample containing muscle and fat. The dimensions of the sample were 60,60 , and $15 \mathrm{~mm}$ along the $x, y$, and $z$ directions, respectively. The fat at the center of the sample was a $15-\mathrm{mm}$ tall cylinder with a diameter of $16 \mathrm{~mm}$. Figure 5(a) shows a two-dimensional image based on the peak amplitude of the acoustic signals generated at the bottom surface of the sample. Figure 5(b) shows a onedimensional image along the horizontal line across the center of the two-dimensional image. A good soft tissue contrast was observed, where the peak acoustic amplitude from the muscle was approximately six times as much as that from the fat.

Since the broad $(\sim 2.2 \mu \mathrm{s})$ microwave pulses and the low-frequency $(1 \mathrm{MHz})$ ultrasonic transducer were used for the experiments shown in Figs. 4 and 5, the lateral resolution was not very good. If the microwave pulses are shortened and the bandwidth of the ultrasonic transducer is broadened, the frequency of the received acoustic signal would be increased leading to improved resolution. However, the key point of these two experiments was to study the contrast.

When a microwave pulse illuminated a sample and deposited energy into the sample, acoustic signals were generated wherever microwave energy was absorbed. The ultrasonic transducer detected the time of arrival signal of the

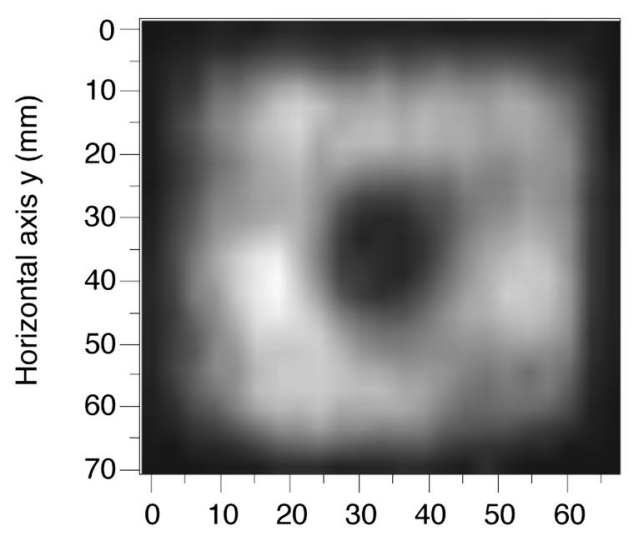

Horizontal axis $\times(\mathrm{mm})$

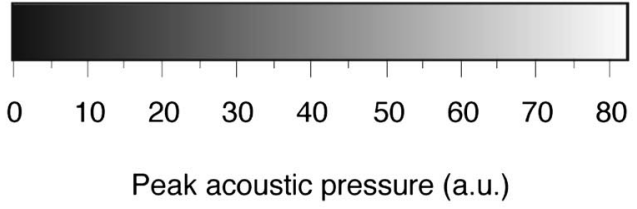

(a)

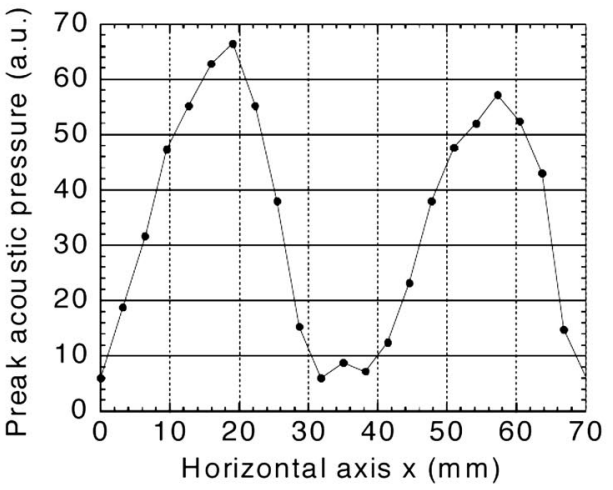

(b)

FIG. 5. (a) Two-dimensional surface image of a tissue sample by use of MIAI. The dark center of the image corresponded to the fat, the bright portion in the image corresponded to the muscle, and the outer black portion corresponded to the mineral oil background. (b) One-dimensional image along the horizontal line across the center of the two-dimensional image in (a)

acoustic sources. The distance between the acoustic sources and the transducer was calculated by multiplying the time of arrival and the speed of sound in the medium. Therefore, a time-domain signal could be converted into a onedimensional image along the transducer axis ( $z$ axis). Scanning the transducer across the sample along the $x$ or $y$ axis would yield a two-dimensional cross-sectional image of the sample in the $x-z$ or $y-z$ plane.

Figure 6 shows a typical acoustic signal from a slice of fat tissue as a function of the relative time of arrival. The first pulse near $5 \mu$ s came from the top surface of the fat tissue. The second pulse near $10 \mu \mathrm{s}$ originated from the bottom surface of the fat tissue. The third pulse near $15 \mu \mathrm{s}$ was from the downward propagating acoustic wave originated from the top surface but reflected by the bottom surface of the fat 


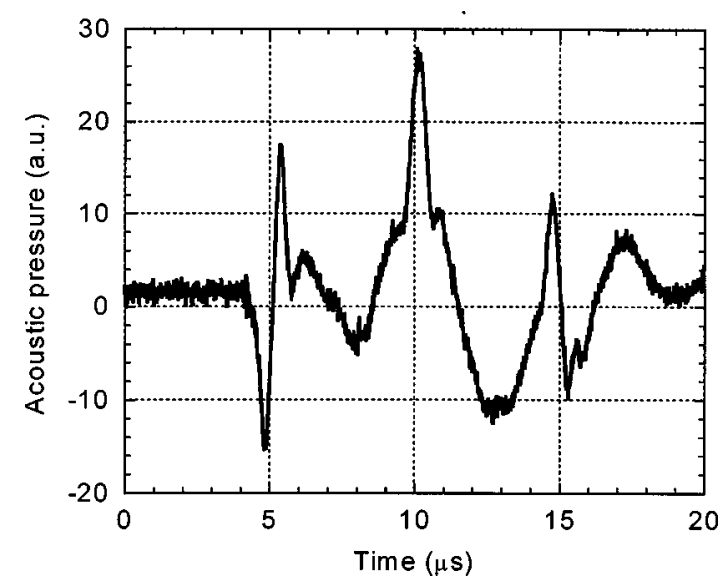

FIG. 6. Sample waveform of microwave-induced acoustic pressure in an 8-mm-thick fat tissue.

tissue. Because the thickness of the sample was $8 \mathrm{~mm}$ and the speed of sound was $\sim 1.5 \mathrm{~mm} / \mu \mathrm{s}$, the expected time interval between the adjacent pulses should be $\sim 5.3 \mu \mathrm{s}$, which matched the observed values very well. The ultrasonic transducer had a central frequency of $3.5 \mathrm{MHz}$ and a 6-dB bandwidth of $2.5 \mathrm{MHz}$. Therefore, the transducer acted as a band-pass filter and rejected the low-frequency acoustic signal originated between the two tissue boundaries.

The sample was translated along the $x$ axis with a step size of $2.6 \mathrm{~mm}$. At each step, the time-domain signal similar to that in Fig. 6 was recorded, which revealed the tissue structure along the $z$ axis. Two-dimensional $x-z$ crosssectional images were then formed by combining the recorded signals at all of the steps, where the negative portion of the signals was set to zero. Three different tissue samples were imaged. Figure 7(a) shows the image of an 8-mm-thick fat slab. The top and bottom surfaces were clearly visible. Figure 7(b) shows the image of an 8-mm-thick fat slab with a U-shaped ditch in the middle. The ditch had a width of 20 $\mathrm{mm}$ and a depth of $5 \mathrm{~mm}$. The observed maximum thickness and minimum thickness in the image were 8 and $3 \mathrm{~mm}$, respectively, which agreed with the actual size of the tissue sample. Figure 7(c) shows the image of a similar 8-mm-thick fat slab with a U-shaped ditch in the middle filled with muscle. The bottom surface of the muscle was visible, but the top surface was invisible because of the high attenuation of microwave at $9.4 \mathrm{GHz}$ in muscle. Microwave with lower frequencies would be necessary to image thicker tissue samples because of the deeper penetration depth of microwave at lower frequencies.

The axial resolution along the $z$ axis was determined by the pulse width of the microwave and the bandwidth of the ultrasonic transducer. We observed that the $3.5-\mathrm{MHz}$ ultrasonic transducer yielded better axial resolution than the 1-MHz transducer. For the results shown in Figs. 6 and 7, the pulse width of the microwave signal was $0.6 \mu \mathrm{s}$, and the temporal width of the acoustic signal was limited to $\sim 1 \mu \mathrm{s}$. The corresponding axial resolution was approximately 1.5 $\mathrm{mm}$ along the $z$ axis under the present experimental conditions. The lateral resolution perpendicularly to the $z$ axis was determined by the numerical aperture and the bandwidth of

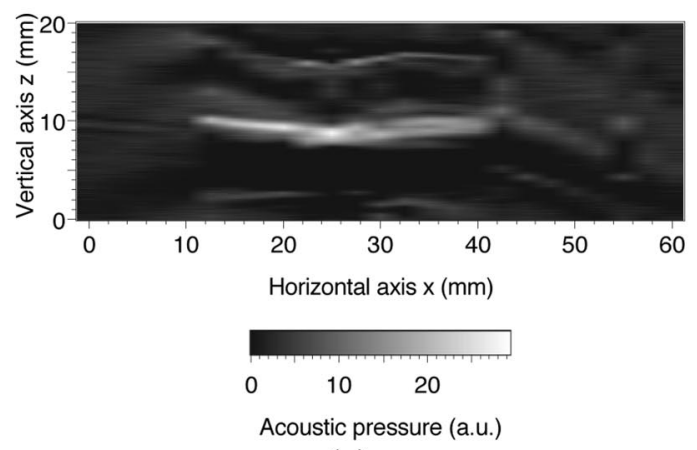

(a)

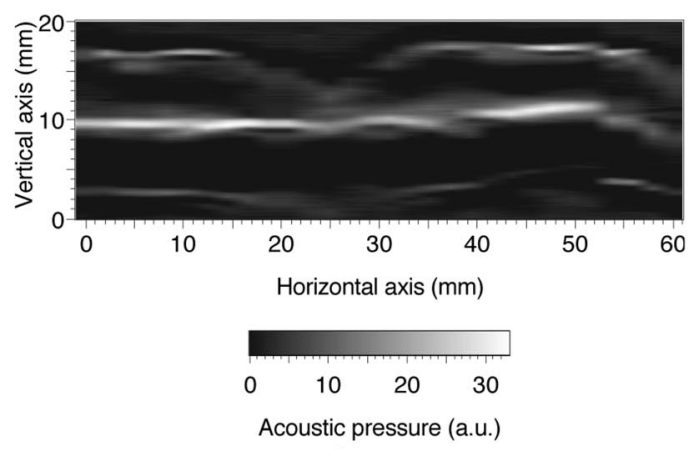

(b)

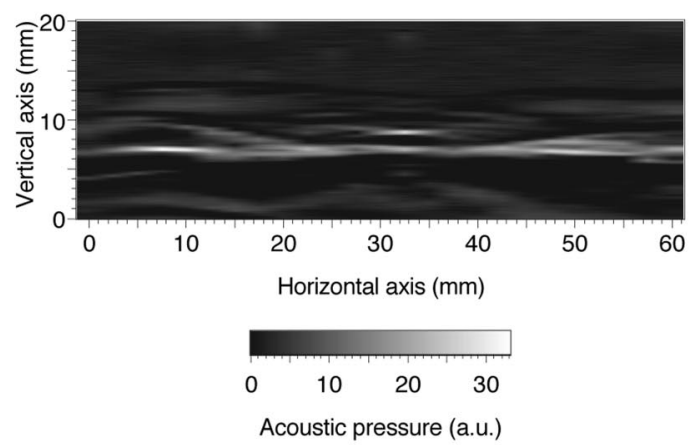

(c)

FIG. 7. Two-dimensional tomographic images of samples with different shapes by use of MIAI. (a) An 8-mm-thick, 30-mm-wide fat tissue slab. (b) An 8-mm-thick fat tissue slab with a U-shaped ditch in the middle, which was $20 \mathrm{~mm}$ long and $5 \mathrm{~mm}$ wide. (c) A similar fat tissue slab as in (b) but having a ditch filled with muscle.

the ultrasonic transducer and by the pulse width of the microwave as well. The lateral resolution of our current setup was $\sim 10 \mathrm{~mm}$ and was to be improved by optimizing the parameters of the ultrasonic transducer and the microwave transmitter. Synthetic aperture technique may be used to further improve the lateral resolution. ${ }^{22}$

\section{DISCUSSION}

Our experimental results demonstrated that microwaveinduced acoustic pressure was proportional to the intensity of the incident microwave. The difference in dielectric constant between different tissue types should provide good imaging contrast in MIAI, which is a different contrast mechanism from that in the conventional ultrasound imaging. We showed that the MIAI technique was able to generate tomog- 
raphic images of biological tissues. The axial resolution along the ultrasonic axis depended on the pulse width of the microwave signal and the bandwidth of the ultrasonic transducer. Combining microwave and ultrasound overcame the resolution problem and the reconstruction problem in the purely microwave imaging.

In the future studies, we plan to lower the microwave frequency and shorten the pulse width to image thicker tissue samples with improved resolution. This technique may also be applied in measurements of the dielectric constants of other materials or of the SAR patterns of electromagnetic fields.

\section{ACKNOWLEDGMENTS}

This project was sponsored in part by the National Institutes of Health Grant Nos. R29 CA68562 and R01 CA71980 and by the National Science Foundation Grant No. BES9734491.

${ }^{1}$ Medical Applications of Microwave Imaging, edited by L. E. Larsen and J. H. Jacobi (IEEE, Piscataway, NJ, 1986).

${ }^{2}$ J. C. Lin, Proc. IEEE 73, 374 (1985).

${ }^{3}$ S. Caorsi, A. Frattoni, G. L. Gragnani, E. Nortino, and M. Pastorino, Med. Biol. Eng. Comput. 29, NS37 (1991).

${ }^{4}$ M. S. Hawley, A. Broquetas, L. Jofre, J. C. Bolomey, and G. Gaboriaud, J. Biomed. Eng. 13, 197 (1991).

${ }^{5}$ P. M. Meaney, K. D. Paulsen, and J. T. Chang, IEEE Trans. Microwave Theory Tech. 46, 31 (1998).

${ }^{6}$ F. Caspers and J. Conway, Proceedings of the 12th European Microwave Conference, 1982, pp. 565-568.
${ }^{7}$ J. L. Su and J. C. Lin, IEEE Trans. Biomed. Eng. 43, 178 (1987).

${ }^{8}$ J. S. K. Wan, Rev. Chem. Intermed. 19, 147 (1993).

${ }^{9}$ T. Bowen, L. Nasoni, A. E. Pifer, and G. H. Sembrosk, Proc. IEEE U1trasonics Symposium 2, 823 (1981).

${ }^{10}$ R. G. Olsen, in Acoustic Imaging, edited by J. P. Powers (Plenum, New York, 1982), pp. 53-59.

${ }^{11}$ R. G. Olsen and J. C. Lin, Bioelectromagnetics (N.Y.) 4, 397 (1983).

${ }^{12}$ J. C. Lin and K. H. Chan, IEEE Trans. Microwave Theory Tech. 32, 854 (1984).

${ }^{13}$ R. L. Nasoni, G. A. Evanoff, Jr., P. G. Halverson, and T. Bowen, Proc. IEEE Ultrasonics Symposium 5, 633 (1984).

${ }^{14}$ K. H. Chan and J. C. Lin, Proceedings of Engineering in Medicine and Biology Society 10th Annual International Conference, 1988, pp. 445446 .

${ }^{15}$ R. A. Kruger, D. R. Reinecke, and G. A. Kruger, Medical Physics (to be published).

${ }^{16}$ R. A. Kruger, K. K. Kopecky, A. M. Aisen, D. R. Reinecke, G. A. Kruger, and W. L. Kiser, Jr., Radiology 211, 275 (1999).

${ }^{17}$ R. A. Kruger, W. L. Kiser, Jr., D. R. Reinecke, and G. A. Kruger, presented at SPIE Conference on Physics of Medical Imaging 3659-43, 20-26 February, 1999.

${ }^{18}$ W. L. Kiser, Jr. and R. A. Kruger, presented at SPIE Conference on Physics of Medical Imaging 3659-96, 20-26 February, 1999.

${ }^{19}$ R. A. Kruger and P. Liu, Med. Phys. 21, 1179 (1994).

${ }^{20}$ R. A. Kruger, P. Liu, Y. R. Fang, and C. R. Appledorn, Med. Phys. 22, 1605 (1995).

${ }^{21}$ A. A. Oraevsky, R. Esenaliev, F. K. Tittel, M. R. Ostermeyer, L.-H. Wang, and S. L. Jacques, Laser-Tissue Interaction VII 2681, 277 (1996).

${ }^{22}$ C. G. A. Hoelen, F. F. M. Demul, R. Pongers, and A. Dekker, Opt. Lett. 23, 648 (1998).

${ }^{23}$ C. Gabriel, S. Gabriel, and E. Corthout, Phys. Med. Biol. 41, 2231 (1996).

${ }^{24}$ S. Gabriel, R. W. Lau, and C. Gabriel, Phys. Med. Biol. 41, 2251 (1996).

${ }^{25}$ S. Gabriel, R. W. Lau, and C. Gabriel, Phys. Med. Biol. 41, 2271 (1996).

${ }^{26}$ F. A. Duck, Physical Properties of Tissue: A Comprehensive Reference Book (Academic, London, New York, 1990). 